\title{
Drag reduction characteristics and flow field analysis of textured surface
}

\author{
Qingshun BAI ${ }^{1,{ }^{*}}$, Jinxuan BAI ${ }^{1}$, Xiangpan MENG ${ }^{2}$, Chengcheng $\mathrm{JI}^{1}$, Yingchun LIANG ${ }^{1}$ \\ ${ }^{1}$ School of Mechanical and Electrical Engineering, Harbin Institute of Technology, Harbin 150001, China \\ ${ }^{2}$ Shanghai Satellite Equipment Research Institute, Shanghai 200000, China \\ Received: 12 June 2015 / Revised: 16 October 2015 / Accepted: 12 May 2016 \\ (C) The author(s) 2016. This article is published with open access at Springerlink.com
}

\begin{abstract}
A textured surface with a micro-groove structure exerts a distinct characteristic on drag reduction behavior. The fluid dynamic models of four textured surfaces are constructed in various profile geometries. Computational fluid dynamics is used to study the friction factors and drag reduction properties with various flow speeds on the textured surfaces. The friction coefficient varieties in the interface between the fluid and the textured surface are examined according to the simulation of the four geometries with V-shaped, saw tooth, rectangular, and semi-circular sections. The drag reduction efficiencies decrease with the increase in water velocity while it is less than a certain value. Moreover, the simulation results of the velocity, shear stress, energy, and turbulence effect on the V-shaped groove surface are presented in comparison with those of the smooth surface to illustrate the drag reduction mechanism. The results indicate that the peaks of the V-shaped grooves inhibit the lateral movement of the turbulent flow and generate the secondary vortex, which plays a key role in the impeding momentum exchange, thereby decreasing turbulent bursting intensity and reducing shear stress in the near-wall flow field. The kinetic energy and turbulence analysis shows that the vortex in the near-wall flow field on the textured surface is more stable compared to that on the smooth surface.
\end{abstract}

Keywords: textured surface; drag reduction; micro-groove; computational fluid dynamics (CFD)

\section{Introduction}

The micro-structures fabricated on a mechanical surface show very practical value because of their special functions, such as drag reduction and anti-icing, dustproof, and self-cleaning abilities, among others $[1,2]$. One may intuitively expect that the smallest skin friction can be obtained on a very smooth surface. However, studies on shark skin and its inspired microstructured surfaces show that the textured surface performs better on drag reduction [3]. The drag reduction phenomenon was discovered by Toms in 1948 [4]. The textured surfaces, which are longitudinally fabricated with micro-grooves with the mean flow direction effectively reduce drag. Although

* Corresponding author: Qingshun BAI.

E-mail: qshbai@hit.edu.cn micro-grooves increase the surface area, experiments showed results of dramatic reduction in flow drag when compared with a smooth surface. Rohr and Andersen observed an equivalent drag reduction performance of a riblet made by the $3 \mathrm{M}$ Company. Their results showed drag reduction peaks between $6 \%$ and $9 \%$ at dimensionless units for a groove height $h^{+}=12$ [5]. Neumann studied a V-shaped groove surface attached to a cylinder and obtained $13 \%$ drag reduction rate in a water tunnel experiment [6]. The riblets could hamper the near wall momentum exchange and delay the development of initial turbulent structures.

Further development of this technology needs a deep understanding of the drag reduction mechanism on the textured surface. NASA has investigated the riblet effects since the 1970s. Walsh [7] first studied the turbulent drag reduction features of the microgrooves on the surface. Bechert et al. [8] investigated the 
riblet geometries with respect to a potential reduction in friction. Accordingly, the ratio of the riblet to the riblet width is the determinant factor. In the aspect of a drag reduction experiment, Gruneberger and Hage [9] and Teo and Khoo [10] examined the momentum exchange of turbulent flow in the near wall region. They found that the fierce momentum could reduce the drag reduction performance. The rough surface flow swept over the grooves. Moreover, the contact area was reduced in comparison with the smooth surface flow, thereby improving the tribological performance [11]. The turbulent characteristics on the textured surface were also investigated in terms of a semi-circular riblet surface [12]. The latest experiments were performed by Aljallis et al. to test the performance of superhydrophobic coated aluminum plates [13]. Most of the stream vortices, which frequently interacted with the riblet tips, were considered to stay above the riblets in the case of drag decrease. The riblet tips impeded the spanwise movement of the streamwise vortices and induced a secondary vortex. Moreover, the velocity fluctuations and turbulent kinetic energy near the riblet surface were lower than those over the smooth surface. Many attempts have been made to investigate the fluid drag mechanisms on the bioinspired structured surface. However, the entirety of the phenomena is not yet fully explained [14].

The secondary vortex is considered to weaken the low-speed fluid ejection from the near-wall region, which consequently impedes the momentum exchange and turbulent bursting intensity. It can also hinder the down-sweeping movement from the high-speed to the low-speed region with a consequent shear stress reduction on the solid wall, thereby stabilizing a lowspeed streak in the valley $[15,16]$. However, the drag reduction mechanisms are not fully understood in terms of velocity, stress, and energy characteristics.

The drag reduction characteristics of the textured surface are presented in this article in terms of the profile geometries and friction coefficient. The background and state-of-the-art research are first critically reviewed. The modeling and computational conditions are illustrated in Section 2. The fluid dynamic model (FDM) is built based on grid optimization, boundary setting, and turbulence model applications. The friction coefficients are researched and analyzed in Section 3.
The drag reduction mechanism is presented with a detailed analysis on the flow field in Section 4 . Conclusions are then drawn in light of the section types and drag reduction mechanism.

\section{Modeling on the metal textured surface}

\subsection{Geometrical parameters of the micro-groove structure}

The simulation models of the textured surfaces with four typical sections (i.e., V-shaped, saw tooth, rectangular, and semi-circular) are built. The feature dimension of all the models is $30 \mu \mathrm{m}$ (Fig. 1). The computational domain models are built in the commercial preprocessor, Gambit, and solved in the software package, Fluent.

The FDM of the four kinds of groove surfaces is proposed in this section. The simulation accuracy is decided by the grid quality. The mesh generation with a fine grid has been adopted in the computational domain near the smooth and groove surfaces because the present study most focuses on the turbulence structure of the top and bottom surfaces. Accordingly, the mesh size gradually increases to the center section. Unstructured triangular grids are applied to the computational domains of the 3D geometrical models to achieve good grid quality (Fig. 2). The Gambit-size function is used to calculate the mesh structure size and capture the flow characteristics during the meshing process. The grid optimization, boundary setting, and turbulence effect are then set to keep the reliable accuracy of the water flow simulation. The size function can let the grids changing from small to big order in the direction of the up-bottom surfaces to the flow (a)
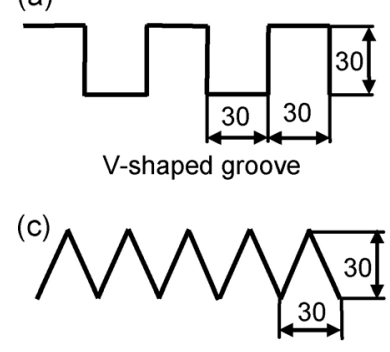

Rectangle groove (b)

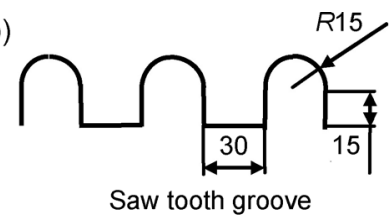

(d)

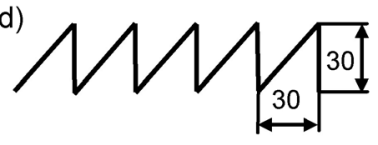

Semi-circular groove
Fig. 1 Geometrical cross-sections of the textured surface. 
(a)

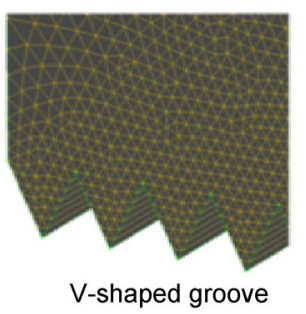

(c)

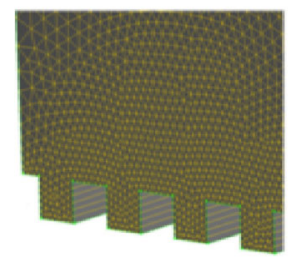

Rectangular groove (b)

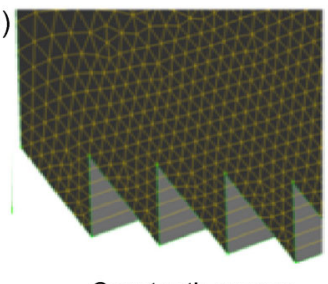

Saw tooth groove

(d)

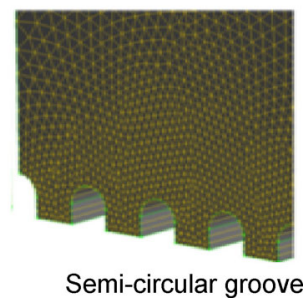

Fig. 2 Modeling of the flow field computational domain.

center. The groove calculation region is set as $0.24 \mathrm{~mm} \times$ $0.43 \mathrm{~mm}$. This kind of meshing method can balance the simulation precision and computational efficiency. The meshed model is checked and adapted to obtain the convergence and consistence results and to affirm that the mesh density cannot affect the calculation results.

\subsection{Boundary conditions and computational parameters}

As regards the turbulent simulation in the research, the Reynolds stress model (RSM), with its strict consideration of vortex flow and complex surface, can be suitable for the modeling process. Therefore, the model adopts the RSM for the simulation. The boundary conditions in the model are illustrated in Fig. 3, with the V-shaped groove surface as an example. The simulation model can be assumed as one part of the limitless flow field. Therefore, the computational domain can be set as the periodic boundary in the two sides. The top side of the computational domain is the smooth surface, while the bottom side acts as the textured surface. Both the top and bottom sides are set as slip-free "wall" sides. The boundary condition settings in the other three types of textured surfaces are the same with the V-shaped groove surface. A coordinate system is set in the model shown in Fig. 3 to conveniently denote the various directions. The $X$ direction represents the transverse direction. The $Y$ direction is the vertical direction of the textured surface. The front and end sides of the $Z$ direction are

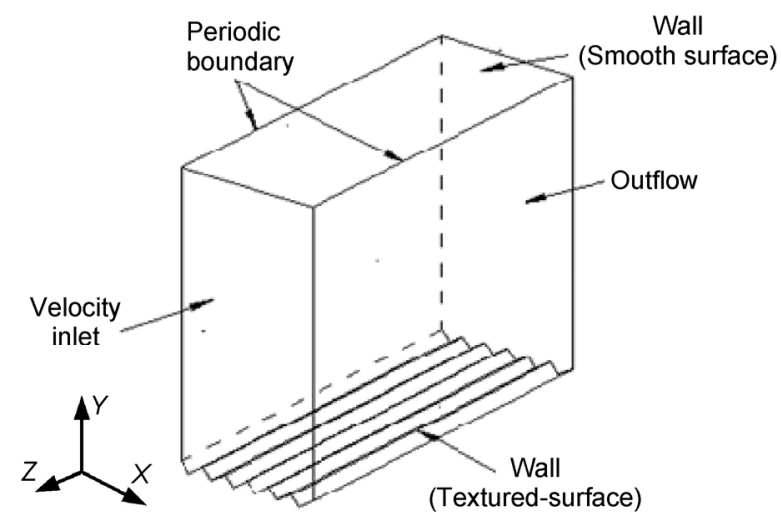

Fig. 3 Boundary condition of the computational domain.

the velocity inlet and outflow side, respectively. The drag reduction rate is presented in Eq. (1) as follows:

$$
\eta=\frac{C_{\mathrm{f}-\text { smooth }}-C_{\mathrm{f}-\text { groove }}}{C_{\mathrm{f}-\text { smooth }}} \times 100 \%
$$

where $C_{\mathrm{f} \text {-smooth }}$ and $C_{\mathrm{f} \text {-groove }}$ are the simulation values of the friction coefficient for the smooth and textured surfaces, respectively; $\eta$ is the drag reduction rate of the simulated surface. The flow velocity can be chosen in the range of $38-55 \mathrm{~m} / \mathrm{s}$, which is thought to cause less error in the simulation. According to NASA's research, the dimensionless units for groove spacing $\left(s^{+}\right)$and height $\left(h^{+}\right)$are chosen as $s^{+}<30$ and $h^{+}<25$, respectively in the simulation that follows. The fluid motion needs to satisfy the continuity and Navier-Stokes Equations, as shown in Eqs. (2) and (3) as follows:

$$
\begin{gathered}
\frac{\partial \rho}{\partial t}+\frac{\partial(\rho u)}{\partial x}+\frac{\partial(\rho v)}{\partial y}+\frac{\partial(\rho w)}{\partial z}=0 \\
\bar{U}_{j} \frac{\partial \bar{U}_{i}}{\partial x_{j}}+\frac{\partial \bar{U}_{i}}{\partial t}=\frac{1}{\rho} \frac{\partial}{\partial x_{j}}\left(\mu \frac{\partial \bar{U}_{i}}{\partial x_{j}}-\rho \bar{u}_{i} \bar{u}_{j}\right)-\frac{1}{\rho} \frac{\partial \bar{P}}{\partial x_{i}}
\end{gathered}
$$

In the present research, the minimum time step is $8 \times 10^{-7} \mathrm{~s}$. The iterations are 25 in the unit time step, which can ensure that the residual error of parameters is less than $1 \times 10^{-5}$. Tables $1-4$ (Appendix A) show all the initial simulation parameters.

\section{Analysis results of the friction coefficients and drag reduction rate}

The friction coefficients from the simulation and 
Prandtl's boundary layer theoretical equation [17] are compared in the simulation of the V-shaped groove textured surface to validate the Renault stress model. The variation curves of the friction coefficient on the smooth and groove surfaces with fluid speed are shown in Fig. 4 to present the textured surface drag reduction. The figure shows that the friction coefficient calculated from the simulation linearly decreases with the speed increase.

Furthermore, the friction coefficient of the textured surface is smaller than that of the smooth surface, which presents good drag reduction characteristics in the fluid speed range in the simulation. About $25.17 \%$ of the maximum drag reduction rate is obtained in this model. The micro-morphology drag reduction can generally be $8 \%-12 \%$. More than $20 \%$ of the drag reduction rate can be achieved on the hydrophobic surface [18]. From our research, it has been proven that the investigated textured surface can present super-hydrophobicity [19]. The drag reduction effect in the simulation can be validated by some results from other researchers under certain conditions [20-22]. The literature review shows that the maximum drag reduction rate of more than $20 \%$ is possible for the hydrophobic, even super-hydrophobic surface. However, the results have a close dependence on the simulation and experiment conditions. The simulation model adopts the no-slip boundary condition. Hence, the drag reduction rate obtained here is mainly determined by the section shape and parameter of the textured surfaces.

Figure 5 shows the simulation results of the other three textured and smooth surfaces. The simulated friction coefficient curve of the rectangular groove is

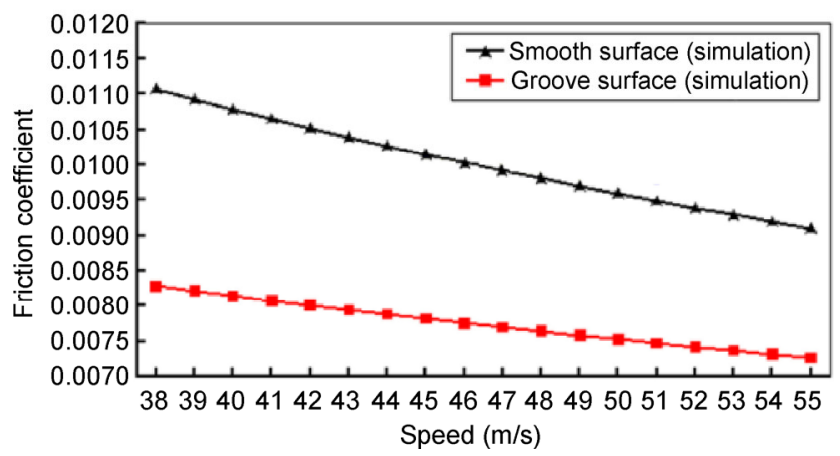

Fig. 4 Friction coefficient of the V-shaped groove textured surface.

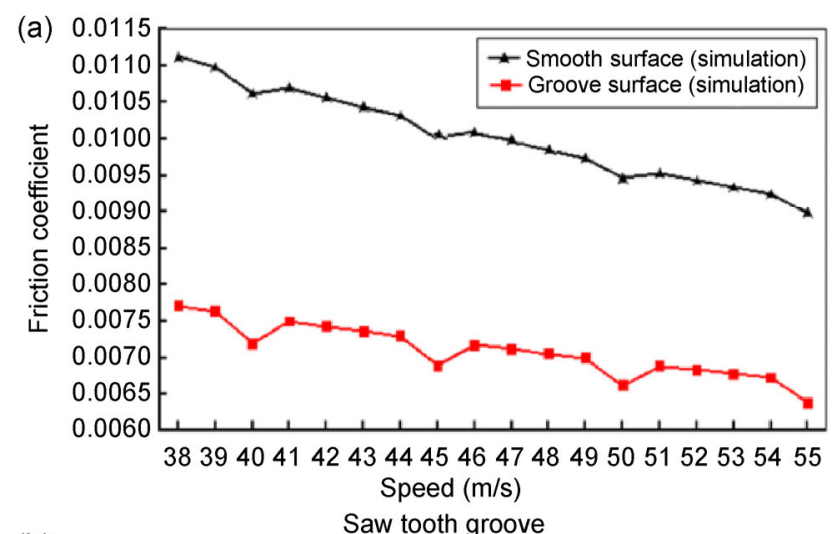

(b)
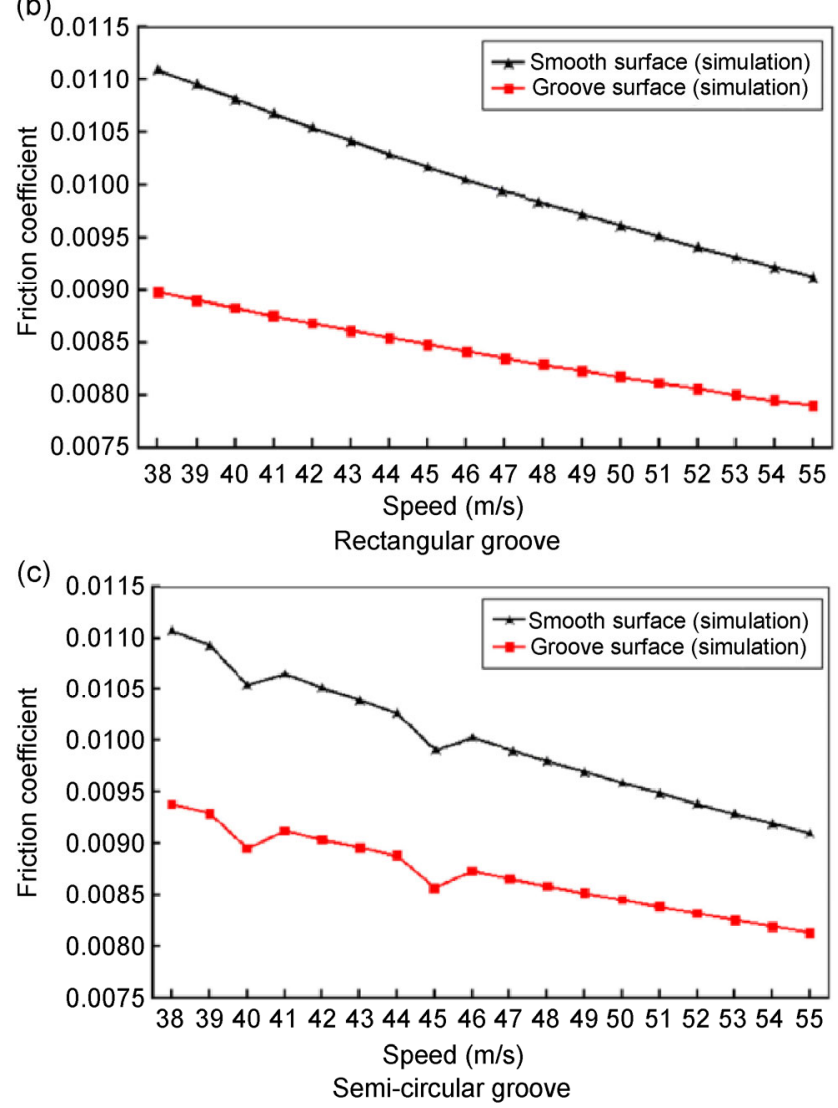

Fig. 5 Friction coefficient of the other three textured surfaces.

similar to that of the V-shaped groove. However, some fluctuations have been observed on the saw tooth and semi-circular groove surfaces. The drag coefficient from the simulation is a little bit larger than the empirical value. The mesh close to the textured and smooth surfaces cannot be refined because of the limitation in the computing ability and efficiency, which leads to the omission of a tiny flow field variation. Furthermore, the ideal simulation conditions and general algorithm may cause the resultant error. 
This result may help choose the good speed condition for better drag reduction.

The drag reduction rates in the four types of textured surface are presented in Fig. 6 to compare the drag reduction characteristics. The drag reduction efficiency is arranged from high to low order as follows: saw tooth groove, V-shaped groove, rectangular groove, and semi-circular groove. No overlap of the drag reduction rate curves in the speed range of simulation has been observed, which can provide guidance for the textured surface application. This result is approximately consistent with that of Henoch [23].

\section{Flow field analysis for the textured and smooth surfaces}

\subsection{Fluid velocity on the textured and smooth surfaces}

The main factor affecting the surface shear stress is the momentum exchange properties in the turbulent layer close to the flow field wall. An analysis on the flow field of solid wall is necessary for an illustrative purpose of the drag reduction mechanism. The fluid velocities of the $\mathrm{V}$-shaped groove and smooth surfaces are simulated under a fluid velocity of $47 \mathrm{~m} / \mathrm{s}$ to minimize the simulation error after many tentative simulations. Figure 7 presents the cloud picture of the velocities of the V-shaped groove and smooth surfaces. Figure 7(a) shows that the speed of the solid-fluid interface of the V-shaped groove surface is zero on the slip-free "wall" side. Figure 7(b) also illustrates

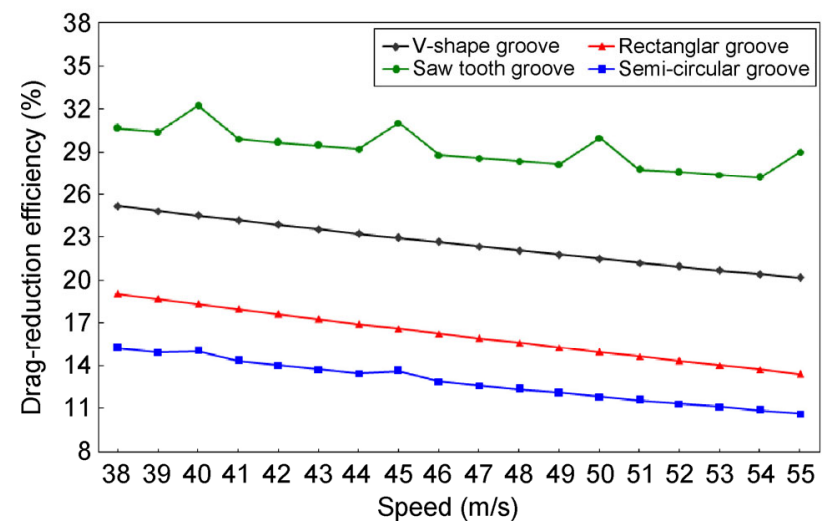

Fig. 6 Drag reduction efficiencies in the four types of textured surfaces. (a)

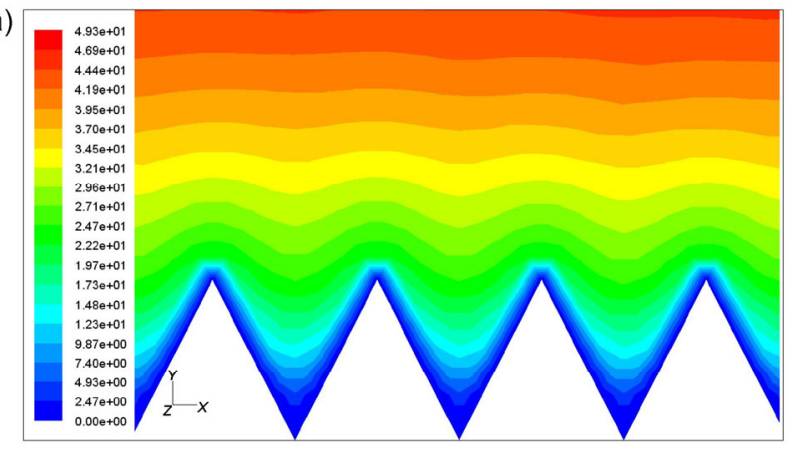

(b)

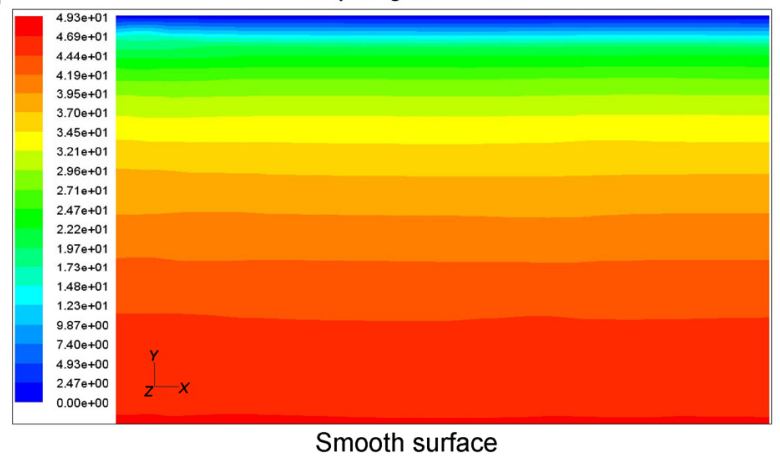

Fig. 7 Cloud picture for the velocities of the V-shaped groove and the smooth surfaces.

that the speed of the solid-fluid interface on the top smooth surface is zero. The velocity gradient of the V-shaped groove surface in the near-wall region is obviously lower than that in the smooth surface. Furthermore, the V-shaped groove surface has a wider bandwidth of the low-speed streak. In other words, the low-speed streak in the near-wall flow field can decrease the direct influence of the high-speed flow in the upper layer on the solid wall, thereby decreasing the flow resistance.

Figure 8 shows the variation curves of the mean velocity gradient and the transverse ( $X$ direction) velocity on the $\mathrm{V}$-shaped groove and the smooth surfaces. The velocity variation close to the wall on the textured surface is slightly slower than that on the smooth surface. The result is consistent with the velocity gradient mechanism shown in Fig. 7. The lower shear stress can be achieved under this condition. Furthermore, the maximum value of the $X$ direction velocity on the textured surface is significantly less than that on the smooth surface in view of the left and right sides of Fig. 8(b). This result indicates that the groove can inhibit the lateral movement of the turbulent flow, thereby stabilizing the near-wall flow 

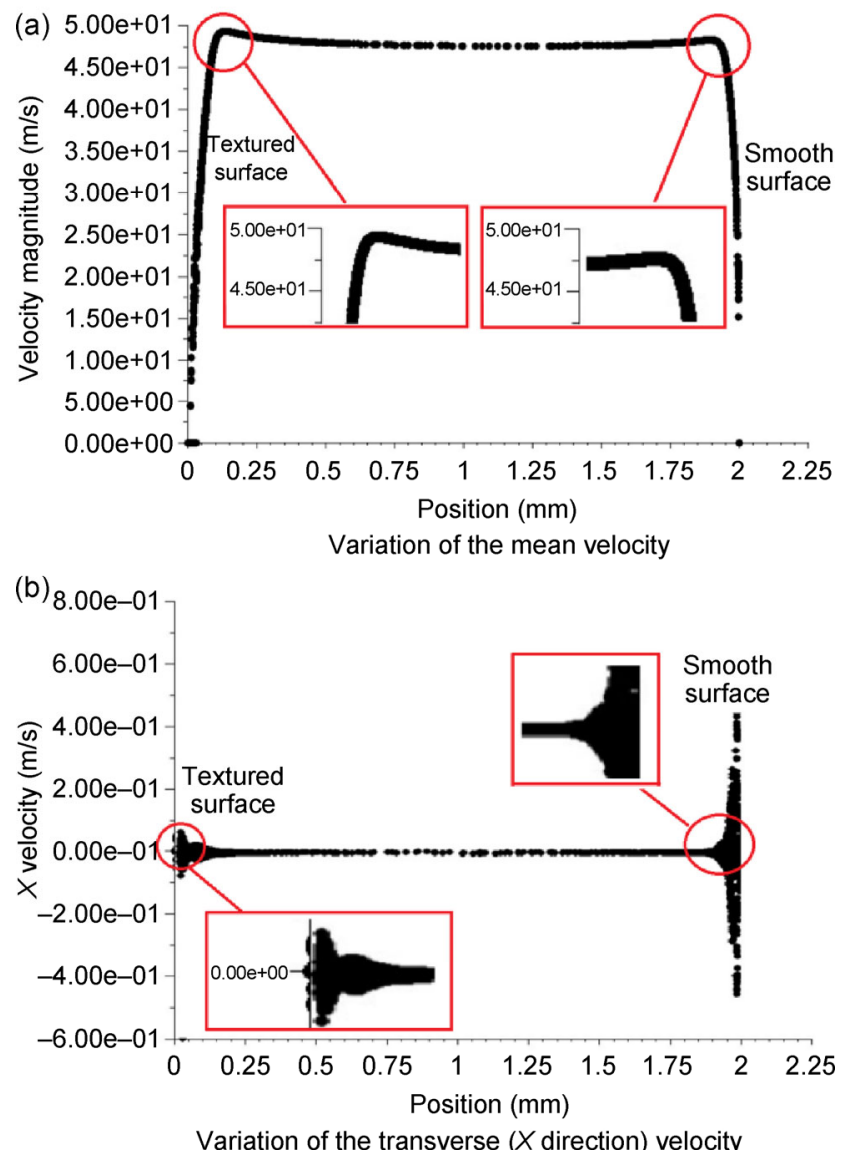

Fig. 8 Velocities of the textured and smooth surfaces.

field. In light of the velocity variations, the fundamental drag reduction mechanism on the micro-grooved surface is that the flow resistance along the flow direction is lower than that on the lateral direction.

Figure 9 shows the velocity simulation of the $V$-shaped groove surface on the $X$ and $Y$ directions. Noticeably, the two deflecting secondary vortex flows occur in opposite directions (Fig. 9(a)). Figure 9(b) shows the low-speed elliptical flow field in the groove valley. In other words, the micro-structure on textured surface can inhibit the ascending movement from the bottom surface, which consequently impedes the momentum exchange. The simulations in the $\mathrm{Z}$ direction velocity also present the general result that the smallest velocity generally appears near the solid surface. The possibility of turbulent bursting is also decreased in this condition. Moreover, the vortex results can prevent the down-sweeping movement from the upper high-speed flow and keep the relative stability of the low-speed flow in the near-wall flow (a)

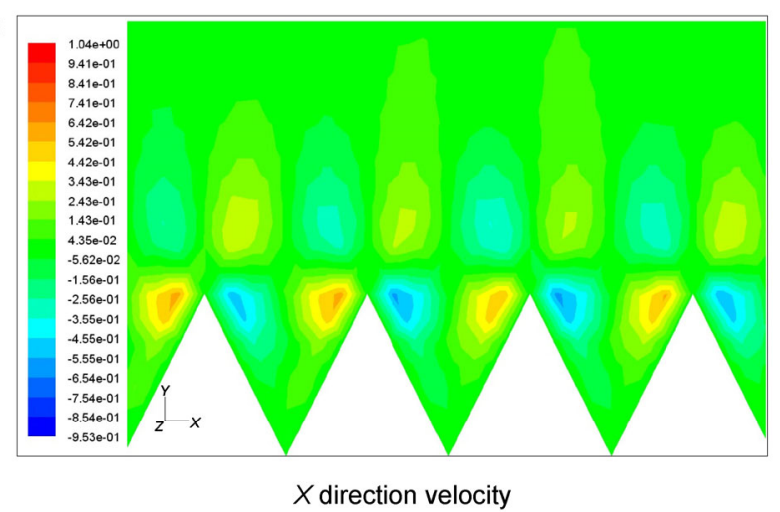

(b)

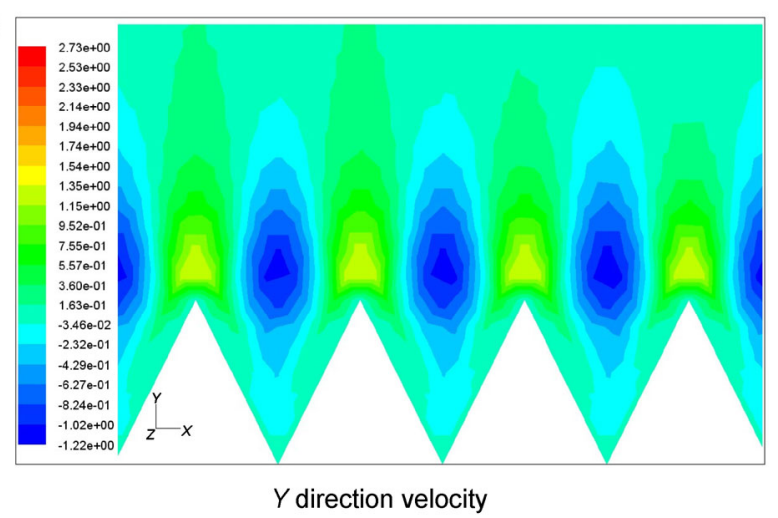

Fig. 9 Velocity distribution of the V-shaped groove surface.

field. The results agree with the hypothesis of the secondary vortex functions $[11,24]$.

\subsection{Shear stress on the textured and smooth surfaces}

Figure 10 shows the shear stress on the textured and smooth surfaces. The picture of the textured surface illustrates a dramatic shear stress reduction in the valleys. In contrast with the smooth surface, the shear stress is high on the peaks of the V-shaped groove. The peaks play a key role in limiting the lateral fluid movement. Therefore, seeing such a high shear stress on the peak is reasonable. However, the calculation shows that the average shear stress on the whole textured surface is less than that on the smooth surface. More areas of the textured surface are distributed in the low shear stress region. The average shear stress in the interface may affect the surface friction and drag behavior.

Generally, the shear stress should be similar in different positions on the smooth surface. Noticeably, an unequal stress exists in the center of Fig. 10(b). The flow field variation may induce the stress 
(a)

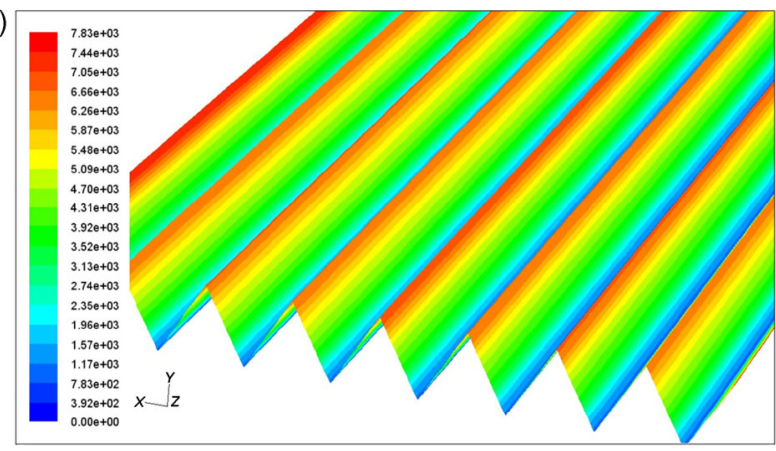

V-shaped groove surface

(b)

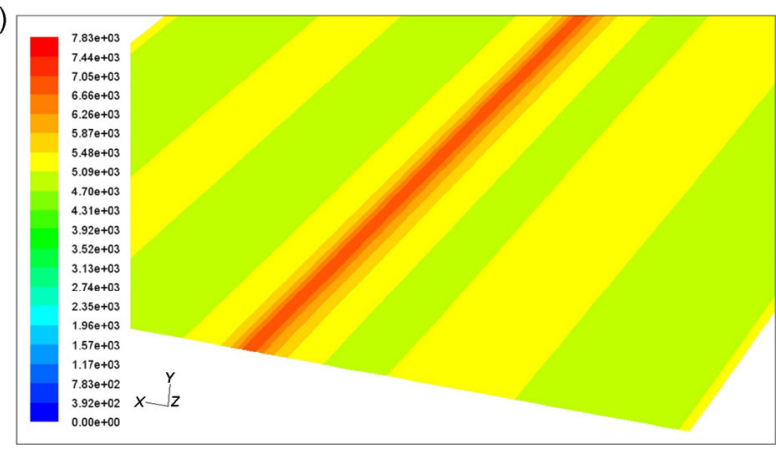

Smooth surface

Fig. 10 Shear stress on the V-shaped groove and smooth surfaces.

fluctuation in different positions. The side wall was designed for the periodic boundary condition. The meshing error in the model may contribute to the difference. The model height was kept large enough to avoid the size effect and the effects on the flow field. The unequal stress cannot be eliminated from the simulation. However, the model and the grid were checked and optimized to assure that the results can be convergent and identical throughout the simulation.

\subsection{Energy on the textured and smooth surfaces}

The kinetic energy in the near-wall flow field was also analyzed to interpret the drag reduction effect. The differences in the turbulent kinetic energy can be noted in the cloud pictures in Fig. 11. The V-shaped groove surface presented a wider width of the lowenergy streak, which meant that the near-wall region of the V-shaped groove surface is more stable than the smooth surface. A semi-circular, low-energy region was also observed on the groove peak, which was an interesting phenomenon showing that sharp peaks can affect the local flow movement. Some regions of lower kinetic energy were found in the textured (a)

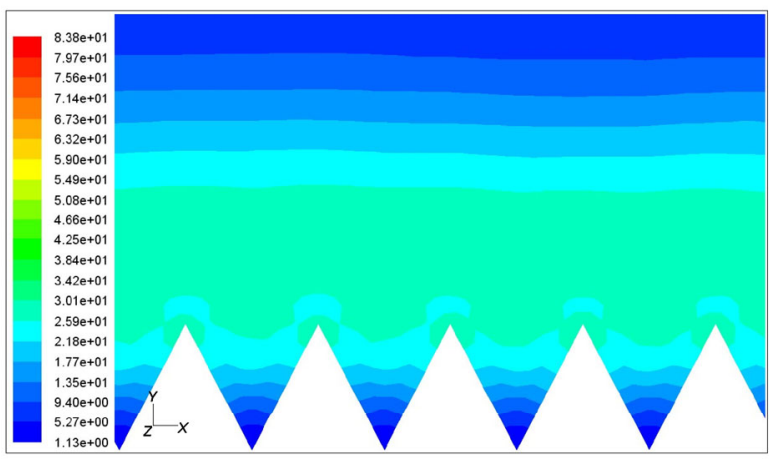

Turbulent kinetic energy on the $\mathrm{V}$-shaped groove surface

(b)

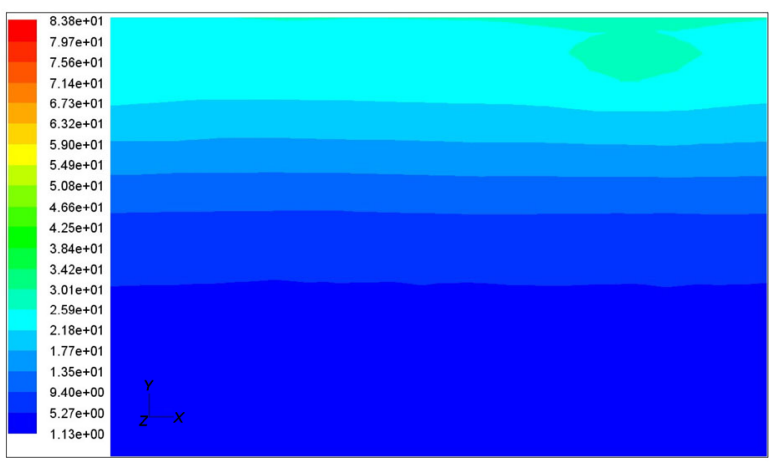

Turbulent kinetic energy on the smooth surface

Fig. 11 Energy on the textured and smooth surfaces.

surface valleys, which was consistent with the preceding velocity variation. The maximum value of the turbulent dissipation rate calculated from the grooved surface was $0.4 \mathrm{~m}^{2} / \mathrm{s}^{2}$, which was $\sim 25 \%$ lower than that from the smooth surface (i.e., $0.525 \mathrm{~m}^{2} / \mathrm{s}^{2}$ ). In other words, there is less momentum exchange and relatively stable flow in the near-wall flow field of the textured surface.

\subsection{Turbulence effect on the textured and smooth surfaces}

The dissipation behavior caused by turbulent bursting in the turbulent boundary layer was the direct reason for energy consumption, which was also a key factor for flow resistance. Therefore, the turbulent, mean swirl, transverse swirl intensities, and Reynolds stress on the textured and smooth surfaces were all measured to investigate the influence of turbulence on drag reduction. The turbulent intensity is usually defined as the ratio of the average velocity to the pulse velocity. It is low-intensity turbulence when the turbulent intensity is lower than or equal to $1 \%$. The high-intensity turbulence is the status of the turbulent 
intensity higher than $10 \%$. Figure 12(a) shows that the maximum turbulent intensity on the textured surface is $1.1 \%$, which is lower than that on the smooth surface (i.e., $1.25 \%$ ). The decreased turbulent intensity can be beneficial for the drag reduction of the textured surface. This turbulence effect on the super-hydrophobic surface can be validated by reference [21]. Moreover, the value was consistent with the experiment and simulation results of previous researchers [10, 25]. Figure 12(b) shows the variations of the mean swirl intensity. In the figure, the peak value of the mean swirl intensity on the textured surface was $5.25 \times 10^{6} 1 / \mathrm{s}$, which was also lower than that on the smooth surface (i.e., $6.75 \times 10^{6} 1 / \mathrm{s}$ ). The flow resistance in the textured surface has been down by nearly $22.2 \%$, which was close to the results obtained by Huo et al. [22]. The reason lies in the combination of the microscale surface morphology and hydrophobicity results in a shear-free interface, which reduced the friction resistance and led to the mean swirl intensity decline. The good hydrophobic
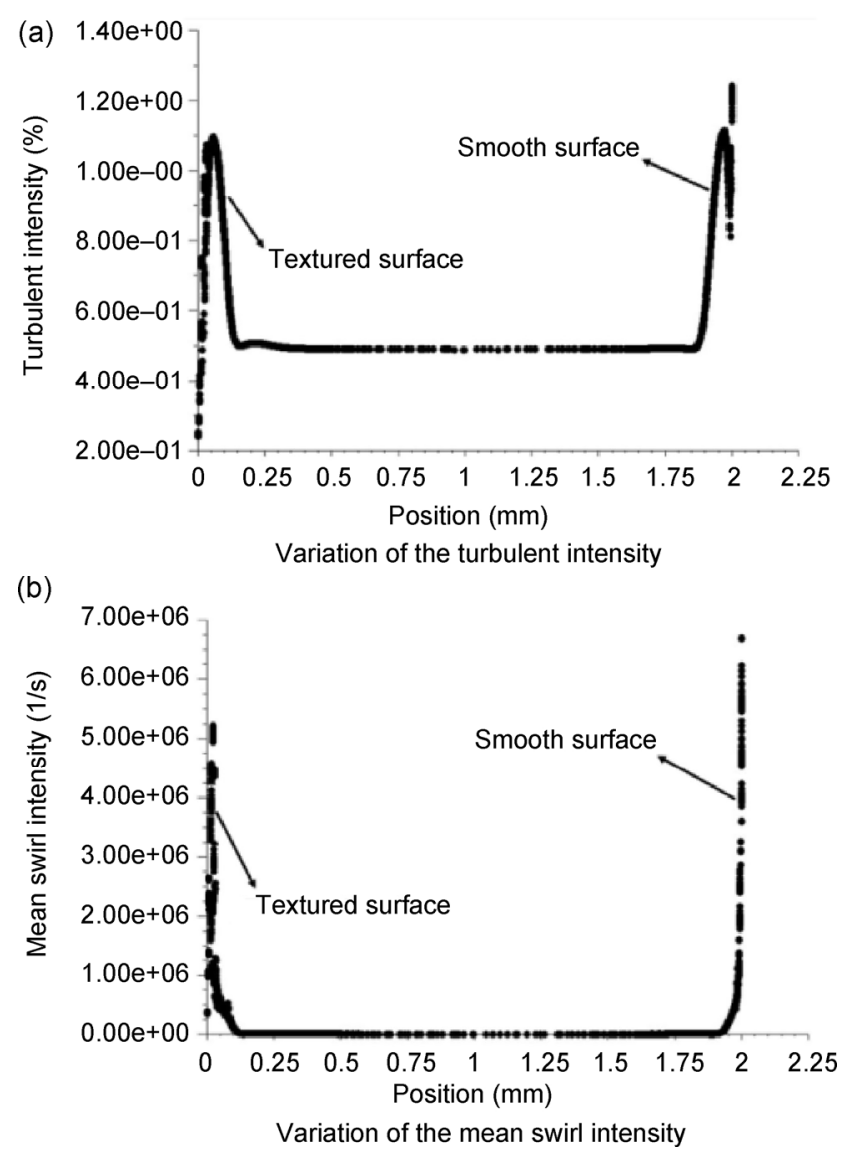

Fig. 12 Turbulence on the textured and smooth surfaces. characteristics were proven from the previous investigation, which can establish the bases for achieving a high drag reduction rate [19]. The results for the Reynolds stress and transverse swirl were analyzed to be less than those on the smooth surface, which meant that the vortex in the near-wall field on the textured surface was more stable. This may decrease the negative effect of turbulent dissipation and shear stress on drag reduction. The stable flow can be beneficial to the drag reduction of the textured surface.

\section{Conclusions}

Four different simulation models of the textured surfaces are presented and analyzed in terms of the drag reduction properties in the fluid field. The drag reduction mechanism is also investigated from the aspect of fluid velocity, shear stress, turbulent kinetic energy, and turbulence effect by taking the V-shaped groove surface as a research object. The following conclusions can be drawn based on this study:

1. The drag reduction efficiencies vary among all the types of textured surfaces simulated, which means that the profile geometries are quite important for optimizing the structural parameters in terms of good drag reduction. The micro-grooves reduce the transverse fluctuation, momentum exchange shear stress, and energy fluctuation. They also stabilize the near-wall flow field. The simulation results show that the V-shaped groove textured surface could obtain the maximum friction coefficient.

2. The maximum value of the $X$ direction velocity on the textured surface is significantly less than that on the smooth surface. A low-speed elliptical flow field in the groove valley can be found. The lowspeed streaks in the near-wall flow field can decrease the direct influence of the upper layer, high-speed flow on the solid wall. The lower shear stress and energy occur in the valley.

3. High shear stress and low-energy, semi-circular regions, which can limit the lateral fluid movement, are found on the V-shaped groove peak. The maximum turbulent and mean swirl intensities on the textured surface are lower than those on the smooth surface, thereby forming a relatively stable flow field. 
4. The secondary vortex is systematically revealed on the textured surface. The effect mechanism on the drag reduction is analyzed and proven in the aspect of fluid velocity, energy, and turbulence effect. The vortex can increase the low speed flow stability in the near-wall flow field. This effect can contribute to the drag reduction characteristics on the textured surface.

\section{Acknowledgments}

This research work was jointly supported by the National Natural Science Foundation of China (No. 51575138) and the State Key Program of National Natural Science Foundation of China (No. 51535003).

\section{Appendix A: Initial simulation parameters}

The simulation velocity has been limited in $38-55 \mathrm{~m} / \mathrm{s}$. The initial simulation parameters are listed in Tables $1-4$, where $m$ is the cross-section mass flow $(\mathrm{kg}) ; u$ is the average incident flow speed $(\mathrm{m} / \mathrm{s})$; Re is the Reynolds number; $k$ is the turbulent kinetic energy $\left(\mathrm{m}^{2} / \mathrm{s}^{2}\right)$; and $\varepsilon$ is the turbulent dissipation rate $\left(\mathrm{m}^{2} / \mathrm{s}^{3}\right)$.

Table 1 Initial parameters of the V-shaped groove.

\begin{tabular}{ccccc}
\hline$m$ & $u$ & $\mathrm{Re}$ & $k$ & $\varepsilon$ \\
\hline 0.0226 & 38 & 18346 & 4.8 & 50324.1 \\
0.0232 & 39 & 18828 & 5.0 & 53875.2 \\
0.0238 & 40 & 19311 & 5.2 & 57577.3 \\
0.0244 & 41 & 19794 & 5.4 & 61433.0 \\
0.0250 & 42 & 20277 & 5.7 & 65444.5 \\
0.0256 & 43 & 20759 & 5.9 & 69614.3 \\
0.0262 & 44 & 21242 & 6.2 & 73944.7 \\
0.0267 & 45 & 21725 & 6.4 & 78438.0 \\
0.0273 & 46 & 22208 & 6.7 & 83096.6 \\
0.0279 & 47 & 22691 & 6.9 & 87922.7 \\
0.0285 & 48 & 23173 & 7.2 & 92918.5 \\
0.0291 & 49 & 23656 & 7.4 & 98086.4 \\
0.0297 & 50 & 24139 & 7.7 & 103428.5 \\
0.0303 & 51 & 24622 & 8.0 & 108947.1 \\
0.0309 & 52 & 25104 & 8.2 & 114644.4 \\
0.0315 & 53 & 25587 & 8.5 & 120522.5 \\
0.0321 & 54 & 26070 & 8.8 & 126583.6 \\
0.0327 & 55 & 26553 & 9.1 & 132829.9 \\
\hline
\end{tabular}

Table 2 Initial parameters of the saw tooth groove.

\begin{tabular}{ccccc}
\hline$m$ & $u$ & $\mathrm{Re}$ & $k$ & $\varepsilon$ \\
\hline 0.0226 & 38 & 18145 & 4.8 & 51090.0 \\
0.0232 & 39 & 18623 & 5.0 & 54695.1 \\
0.0238 & 40 & 19100 & 5.2 & 58453.6 \\
0.0244 & 41 & 19578 & 5.5 & 62368.0 \\
0.0250 & 42 & 20055 & 5.7 & 66440.6 \\
0.0256 & 43 & 20533 & 5.9 & 70673.9 \\
0.0262 & 44 & 21010 & 6.2 & 75070.2 \\
0.0267 & 45 & 21488 & 6.4 & 79631.9 \\
0.0273 & 46 & 21965 & 6.7 & 84361.3 \\
0.0279 & 47 & 22443 & 6.9 & 89260.8 \\
0.0285 & 48 & 22920 & 7.2 & 94332.7 \\
0.0291 & 49 & 23398 & 7.5 & 99579.2 \\
0.0297 & 50 & 23875 & 7.7 & 105002.7 \\
0.0303 & 51 & 24353 & 8.0 & 110605.3 \\
0.0309 & 52 & 24830 & 8.3 & 116389.3 \\
0.0315 & 53 & 25308 & 8.6 & 122356.8 \\
0.0321 & 54 & 25785 & 8.8 & 128510.2 \\
0.0327 & 55 & 26263 & 9.1 & 134851.6 \\
\hline & & & &
\end{tabular}

Table 3 Initial parameters of the rectangular groove.

\begin{tabular}{ccccc}
\hline$m$ & $u$ & $\mathrm{Re}$ & $k$ & $E$ \\
\hline 0.0226 & 38 & 18610 & 4.7 & 49342.4 \\
0.0232 & 39 & 19100 & 5.0 & 52824.2 \\
0.0238 & 40 & 19590 & 5.2 & 56454.2 \\
0.0244 & 41 & 20080 & 5.4 & 60234.6 \\
0.0250 & 42 & 20569 & 5.7 & 64167.9 \\
0.0256 & 43 & 21059 & 5.9 & 68256.4 \\
0.0262 & 44 & 21549 & 6.1 & 72502.3 \\
0.0267 & 45 & 22039 & 6.4 & 76908.0 \\
0.0273 & 46 & 22528 & 6.6 & 81475.7 \\
0.0279 & 47 & 23018 & 6.9 & 86207.6 \\
0.0285 & 48 & 23508 & 7.1 & 91106.0 \\
0.0291 & 49 & 23998 & 7.4 & 96173.0 \\
0.0297 & 50 & 24487 & 7.7 & 101410.9 \\
0.0303 & 51 & 24977 & 7.9 & 106821.9 \\
0.0309 & 52 & 25467 & 8.2 & 112408.0 \\
0.0315 & 53 & 25956 & 8.5 & 118171.5 \\
0.0321 & 54 & 26446 & 8.8 & 124114.4 \\
0.0327 & 55 & 26936 & 9.1 & 130238.9 \\
\hline
\end{tabular}


Table 4 Initial parameters of the semi-circular groove.

\begin{tabular}{ccccc}
\hline$m$ & $u$ & $\mathrm{Re}$ & $k$ & $\varepsilon$ \\
\hline 0.0226 & 38 & 18879 & 4.7 & 48380.1 \\
0.0232 & 39 & 19376 & 5.0 & 51793.9 \\
0.0238 & 40 & 19872 & 5.2 & 55353.1 \\
0.0244 & 41 & 20369 & 5.4 & 59059.8 \\
0.0250 & 42 & 20866 & 5.6 & 62916.4 \\
0.0256 & 43 & 21363 & 5.9 & 66925.1 \\
0.0262 & 44 & 21860 & 6.1 & 71088.2 \\
0.0268 & 45 & 22356 & 6.4 & 75408.0 \\
0.0274 & 46 & 22853 & 6.6 & 79886.6 \\
0.0280 & 47 & 23350 & 6.9 & 84526.2 \\
0.0286 & 48 & 23847 & 7.1 & 89329.0 \\
0.0292 & 49 & 24344 & 7.4 & 94297.3 \\
0.0297 & 50 & 24841 & 7.6 & 99433.0 \\
0.0303 & 51 & 25337 & 7.9 & 104738.4 \\
0.0309 & 52 & 25834 & 8.2 & 110215.6 \\
0.0315 & 53 & 26331 & 8.5 & 115866.7 \\
0.0321 & 54 & 26828 & 8.7 & 121693.7 \\
0.0327 & 55 & 27325 & 9.0 & 127698.7 \\
\hline
\end{tabular}

Open Access: The articles published in this journal are distributed under the terms of the Creative Commons Attribution 4.0 International License (http:// creativecommons.org/licenses/by/4.0/), which permits unrestricted use, distribution, and reproduction in any medium, provided you give appropriate credit to the original author(s) and the source, provide a link to the Creative Commons license, and indicate if changes were made.

\section{References}

[1] Yan Y Y, Gao N. Mimicking natural super-hydrophobic surfaces and grasping the wetting process: A review on recent progress in preparing super-hydrophobic surfaces. Adv Colloid Interface 169: 80-105 (2011)

[2] Bruzzone A A G, Costa H L, Lonardo P M, Lucca D A. Advances in engineered surfaces for functional performance. CIRP Annals-Manuf Technol 57: 750-769 (2008)

[3] Gregory D B, Bharat B. Shark skin inspired low-drag microstrurctured surfaces in closed channel flow. J Colloid Interface 393: 384-396 (2013)

[4] Brostow W. Drag reduction in flow: review of applications, mechanism and prediction. $J$ Ind Eng Chem 14: 409-416 (2008)

[5] Rohr J J, Andersen G W. A comparison of the dragreducing benefits of riblets in internal and external flows. Exp Fluids 13: 361-368 (1992)

[6] Neumann D. Drag measurements on v-grooved surfaces on a body of revolution in axial flow. Appl Sci Res 48: 105-114 (1991)

[7] Bushnell D M, Hefner J N. Viscous Drag Reduction in Boundary Layers. American Institute of Aeronautics and Astronautics Inc., 1990.

[8] Bechert D W, Bruse M, Hage W, Hoppe G. Experiments on drag-reducing surfaces and their optimization with an adjustable geometry. J Fluid Mech 338: 59-87 (1997)

[9] Gruneberger R, Hage W. Drag characteristics of longitudinal and transverse riblets at low dimensionless spacings. Exp Fluids 50: 363-373 (2011)

[10] Teo C J, Khoo B C. Flow past super-hydrophobic surfaces containing longitudinal grooves: Effects of interface curvature. Microfluid Nanofluid 9: 499-511 (2010)

[11] Cui J, Li W Z, Lam W H. Numerical investigation on drag reduction with superhydrophobic surfaces by latticeBoltzmann method. Comput Math Appl 61: 3678-3689 (2011)

[12] Lee S J, Lee S H. Flow field analysis of a turbulent boundary layer over a riblet surface. Exp Fluids 30: 153-166 (2001)

[13] Aljallis E, Sarshar M A, Datla R, Sikka V, Jones A, Choi C-H. Experimental study of skin friction drag reduction on superhydrophobic flat plates in high Reynolds number boundary layer flow. Phys Fluids 25: 025103-1-14 (2013)

[14] Bhushan B. Bioinspired structured surfaces. Langmuir 28: 1698-1714 (2012)

[15] Friedmann E. The optimal shape of riblets in the viscous sublayer. J Math Fluid Mech 12: 243-265 (2012)

[16] Muradoglu M, Tasoglu S. A front-tracking method for computational modeling of impact and spreading of viscous droplets on solid walls. Comput Fluids 39: 615-625 (2010)

[17] Erhard P, Etling D, Muller U, Riedel U, Sreenivasan K R, Warnatz J. Prandtl-Essentials of Fluid Mechanics (3rd Edition). Springer-Verlag New York Inc., 2009.

[18] Luo Y, Wang L, Green L, Song K, Wang L, Smith R. Advances of drag-reducing surface technologies in turbulence based on boundary layer control. Journal of Hydrodynamics 27(4): 473-487 (2015)

[19] Bai Q S, Meng X P, Liang Y C, Cheng K. Static and dynamic interface characteristics between functional micro-structure and water. International Journal of Nanomanufacturing 11(1/2): 13-24 (2015) 
[20] Huang Q, Pan G, Wu H, Hu H, Song B. Investigation about drag reduction water tunnel experiment and mechanism of superhydrophobic surface. (in Chinese). Journal of Experiments in Fluid Mechanics 25(5): 21-25 (2011)

[21] Daniello R J, Waterhouse N E, Rothstein J P. Drag reduction in turbulent flows over superhydrophobic surfaces. Phys Fluids 21: 085103 (2009)

[22] Huo S, Yu Z, Li Y, Liu Y, Sun X, Song S. Flow characteristics of water in microchannel with super-hydrophobic surface. (in Chinese). Journal of Chemical Industry and

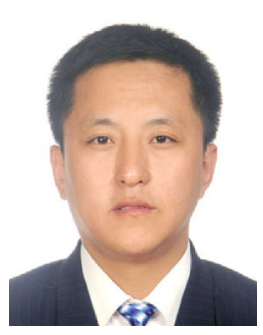

Qingshun BAI. He received his M.S. and Ph.D degrees in mechanical manufacturing \& its automation from Harbin Institute of Technology, China, in 2000 and 2004, respectively. He joined the School of Mechanical and Electrical Engineering at Harbin
Engineering 58(11): 2721-2726 (2007)

[23] Henoch C, Krupenkin T N, Kolodner P, Taylor J A, Hodes M S, Lyons A M, Peguero C, Breue K. Turbulent drag reduction using superhydrophobic surfaces. In the $3 \mathrm{rd}$ AIAA Flow Control Conference, 2006: 1-5.

[24] Bacher E V, Simth C R. Turbulent boundary-layer modification by surface riblets. AIAA J 24: 1382-1385 (1986)

[25] Tsai P, Peters A M, Pirat C, Wessling M, Lammertink R G H, Lohse D. Quantifying effective slip length over micropatterned hydrophobic surfaces. Phys Fluids 21: 112002 (2009)

Institute of Technology from 2004. His current position is an associate professor and the Ph.D candidate supervisor. His research areas cover precision and ultra-precision machining, micro/nano manufacturing technology, and modern design theory and its engineering application. 